\title{
Translation-independent inhibition of mRNA deadenylation during stress in Saccharomyces cerevisiae
}

\author{
VALÉRIE HILGERS, ${ }^{1,2}$ DANIELA TEIXEIRA, ${ }^{1,3}$ and ROY PARKER ${ }^{1}$ \\ ${ }^{1}$ Department of Molecular and Cellular Biology and Howard Hughes Medical Institute, University of Arizona, Tucson, Arizona 85721, USA \\ ${ }^{2}$ Ecole Normale Supérieure, 45, rue d'Ulm, 75005 Paris, France \\ ${ }^{3}$ Instituto de Ciencias Biomedica Abel Salazar, Universidade do Porto, 4050-345 Porto, Portugal
}

\begin{abstract}
Post-transcriptional control mechanisms play an important role in regulating gene expression during cellular responses to stress. For example, many stresses inhibit translation, and at least some stresses inhibit mRNA turnover in yeast and mammalian cells. We show that hyperosmolarity, heat shock, and glucose deprivation stabilize multiple mRNAs in yeast, primarily through inhibition of deadenylation. Although these stresses inhibit translation and promote the movement of mRNAs into P-bodies, we also observed inhibition of deadenylation in cycloheximide-treated cells as well as in a mutant strain where translation initiation is impaired. This argues that inhibition of poly(A)-shortening is independent of the translational state of the mRNAs and can occur when mRNAs are localized in polysomes or are not engaged in translation. Analysis of pan $2 \Delta$ or $c c r 4 \Delta$ strains indicates that stress inhibits the function of both the Ccr4p/Pop2p/Notp and the Pan2p/Pan3p deadenylases. We suggest that under stress, simultaneous repression of translation and deadenylation allows cells to selectively translate mRNAs specific to the stress response, while retaining the majority of the cytoplasmic pool of mRNAs for later reuse and recovery from stress. Moreover, because various cellular stresses also inhibit deadenylation in mammalian cells, this mechanism is likely to be a conserved aspect of the stress response.
\end{abstract}

Keywords: stress; adenylation; yeast; translation; decapping

\section{INTRODUCTION}

The cellular response to environmental stresses requires a set of specific regulatory mechanisms to allow the cell to adapt to the new condition and resume growth. In a wide variety of eukaryotes, including both yeast and mammalian cells, the response to various cellular stresses includes alterations in multiple aspects of mRNA metabolism (for review, see Bond 2006). For example, many stresses lead to a general repression of translation initiation, often by phosphorylation of the alpha subunit of the translation initiation factor eIF2 (for review, see Holcik and Sonenberg 2005).

The cellular response to stress and inhibition of translation initiation also leads to the accumulation of translationally repressed mRNAs within specific cytoplasmic structures (for review, see Anderson and Kedersha 2006). For example, in mammalian cells, nontranslating mRNAs concentrate in

Reprint requests to: Roy Parker, Department of Molecular and Cellular Biology and Howard Hughes Medical Institute, University of Arizona, Tucson, AZ 85721, USA; e-mail: rrparker@u.arizona.edu; fax: (520) 6214524 .

Article published online ahead of print. Article and publication date are at http://www.rnajournal.org/cgi/doi/10.1261/rna.241006. stress granules, cytoplasmic aggregates also containing the 40S ribosomal subunit, the RNA binding proteins TIA and TIA-R, and some translation initiation factors. In yeast, and to a lesser degree in mammalian cells, stress-induced inhibition of protein synthesis causes accumulation of translationally repressed mRNAs in P-bodies. P-bodies are conserved cytoplasmic foci containing various proteins involved in translation repression and mRNA decay, including the mRNA decapping enzyme and the $5^{\prime}$ to $3^{\prime}$ exonuclease Xrnlp (Anderson and Kedersha 2006). While first described as sites of mRNA decapping and $5^{\prime}$ to $3^{\prime}$ exonucleolytic degradation (Sheth and Parker 2003; Cougot et al. 2004), Pbodies have recently been shown to function in conventional and miRNA-mediated translational control as well as mRNA storage (Brengues et al. 2005; Coller and Parker 2005; Liu et al. 2005; Sen and Blau 2005; Teixeira et al. 2005). This stressdependent targeting of mRNAs to P-bodies suggests that the cellular response to stress will also include modulation of the rates of mRNA decay.

Several studies have suggested that mRNA decay rates can be altered in response to various stresses. For example, response to UV-B exposure leads to stabilization of many short-lived mRNAs in mammalian cells (Gowrishankar 
et al. 2006). Conversely, in yeast, mild heat shock promotes rapid degradation of mRNAs encoding the ribosomal proteins (Herruer et al. 1988), while glucose starvation, amino acid starvation, or sugar-induced osmotic stress causes stabilization of at least some yeast mRNAs (Jona et al. 2000; Bénard 2004; Greatrix and van Vuuren 2006). How these changes in mRNA stability are related to the corresponding changes in translation that are occurring during the same stress response is not known.

Another unresolved issue is the mechanism by which stress alters the degradation of eukaryotic mRNAs. In eukaryotic cells, two general pathways are responsible for the decay of cytoplasmic transcripts. Both pathways begin with the shortening of the $3^{\prime}$ poly $(\mathrm{A})$ tail in a process referred to as deadenylation (for review, see Parker and Song 2004). Based on work in yeast cells, deadenylation is primarily catalyzed by the Ccr4p/Pop2p/Notp complex, although a second deadenylase consisting of Pan $2 p$ and Pan3p also contributes to cytoplasmic mRNA deadenylation (Daugeron et al. 2001; Tucker et al. 2001). Following deadenylation, the body of the transcript can be degraded $3^{\prime}$ to $5^{\prime}$ by the cytoplasmic exosome, a complex of several $3^{\prime}$ to $5^{\prime}$ exoribonucleases in eukaryotic cells. Alternatively, and more commonly at least in yeast, the mRNA is decapped by the Dcp1p/Dcp2p decapping enzyme and then degraded $5^{\prime}$ to $3^{\prime}$ by the exoribonuclease Xrn1p. Recently, it has been shown that exposure to UV-B light, hydrogen peroxide, heat shock, and osmotic shock leads to the inhibition of deadenylation on several different specific mRNAs in mammalian cells (Gowrishankar et al. 2006). This suggests that inhibition of deadenylation might be an important aspect of the stress response. Consistent with that possibility, it has been observed that glucose deprivation of yeast cells leads to a global persistence of poly(A) tails, although whether this is actually due to changes in deadenylation of specific mRNAs was not addressed (Jona et al. 2000).

In this work, we examine how different stresses affect the degradation of specific yeast mRNAs, and how these effects are related to the general translational repression that accompanies stress exposure. We observe that multiple stresses stabilize mRNAs in yeast cells, primarily through the inhibition of deadenylation. The stress-dependent inhibition of deadenylation is also seen in cycloheximide-treated cells as well as in a mutant strain where translation initiation is impaired, thus arguing that the inhibition of poly(A)-shortening occurs independent of whether the mRNA is associated with ribosomes. Analysis of pan $2 \Delta$ or $c c r 4 \Delta$ strains indicates that stress inhibits the function of both the Ccr4p/Pop2p/ Notp complex and the Pan2p/Pan3p deadenylases. Based on these observations, we suggest that the simultaneous repression of translation and deadenylation during stress may be functionally important in preventing the degradation of mRNAs that normally occurs with decreased translation. Thus, during a stress response, this combination of effects would allow cells to selectively translate mRNAs specific to the stress response and still retain the majority of the cytoplasmic pool of mRNAs for later reuse and recovery from stress.

\section{RESULTS}

\section{Different stresses affect the turnover of the MFA2 mRNA}

To examine the effect of stress on mRNA turnover, we first monitored the degradation of the MFA2pG reporter transcript in wild-type cells (Decker and Parker 1993). This mRNA is under the control of the GAL1 UAS, and therefore expressed in the presence of galactose. The addition of glucose specifically blocks transcription from this promoter, allowing the measurement of the decay rate of the reporter mRNA.

To monitor the degradation of the MFA2 mRNA, we grew wild-type cells in galactose and shifted the carbon source to glucose with or without a concomitant stress induction. Stress induction was achieved by adding either $1 \mathrm{M} \mathrm{KCl}$ (osmotic stress) or $5 \mathrm{mM}$ DTT (induction of the unfolded protein response) to the resuspension medium or by shifting the cells from $25^{\circ} \mathrm{C}$ to $46^{\circ} \mathrm{C}$ (a robust heat shock). Compared to nonstressed cells (half-life $=5 \mathrm{~min}$ ), the decay of the $M F A 2 p G$ reporter mRNA was substantially reduced when cells were stressed by $1 \mathrm{M} \mathrm{KCl}$ (half-life $=22 \mathrm{~min}$; Fig. $1 \mathrm{~A}$, cf. left and right panels) or heat shock (half-life $=15 \mathrm{~min}$; Fig. 1B). In contrast, no stabilization of the MFA2 mRNA was observed when cells were exposed to DTT treatment for 10 min prior to transcriptional shutoff (Fig. 1D), which induces the unfolded protein response (Travers et al. 2000).

We also examined how MFA2 mRNA decay was affected by glucose deprivation, which is known to induce a stress response in yeast cells (for review, see Bond 2006). Since we could not use a GAL-driven reporter mRNA under conditions of carbon source shifts, we used the temperature sensitive mutation $r p b 1-1$, which leads to an inhibition of transcription following a shift to the nonpermissive temperature of $38^{\circ} \mathrm{C}$ (Herrick et al. 1990). It should be noted that a shift to $38^{\circ} \mathrm{C}$ is not a strong enough heat shock to alter mRNA decay by itself (Fig. 1C, left panel; data not shown). rpb1-1 cells were shifted to media that were prewarmed to $38^{\circ} \mathrm{C}$ and lacked glucose in order to shut off transcription concomitantly with stress induction. We observed that the degradation of the endogenous MFA2 mRNA was slowed in response to glucose deprivation (Fig. 1C), similar to the effects we observed with heat shock and osmotic stress. Together, these results indicate that a variety of cellular stresses can inhibit mRNA degradation.

\section{mRNA stabilization during stress is caused by inhibition of deadenylation}

To determine which step of mRNA decay is affected during stress, we first examined the lengths of the poly $(\mathrm{A})$ tails of the MFA2 mRNA at different time points after transcription 
A

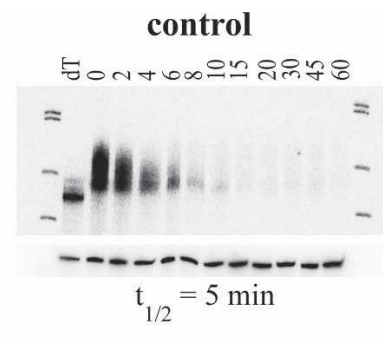

$25^{\circ} \mathrm{C}$

$\mathbf{B}$

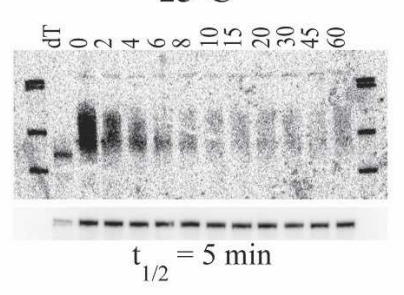

C

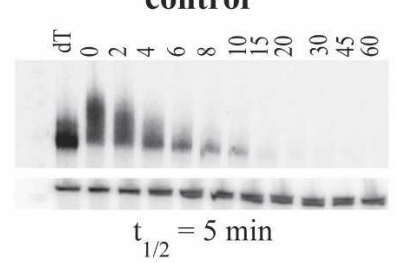

D

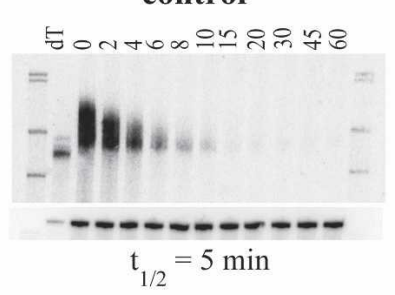

KCl 1M

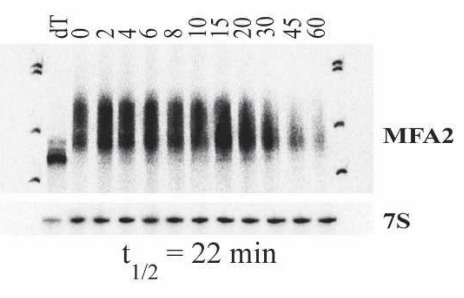

$46^{\circ} \mathrm{C}$

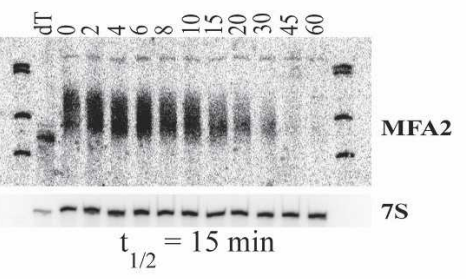

- glucose

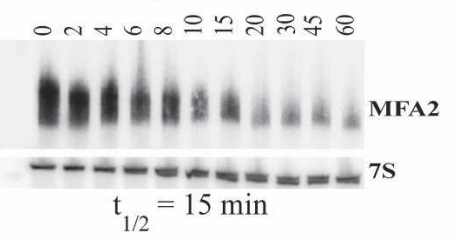

DTT $5 \mathrm{mM}$

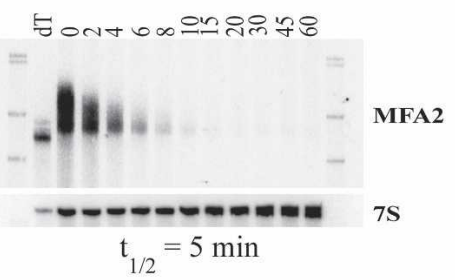

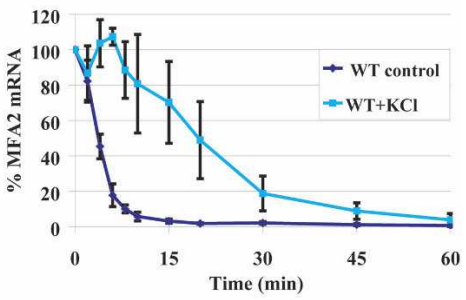
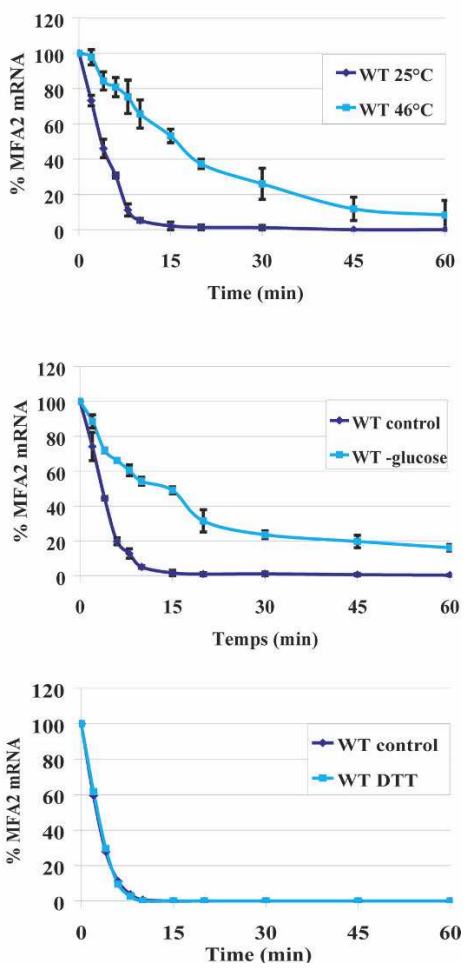

FIGURE 1. Different stresses affect the turnover of the MFA2 mRNA. Shown are polyacrylamide Northern gels examining the decay of MFA2 in wildtype strains $(A, B, D)$ and the rpbl-1 strain $(C)$ without (left panels) and with (right panels) exposure to the indicated stress. Numbers above the lanes represent minutes after transcriptional repression by shifting the carbon source to glucose $(A, B, D)$ or by shifting the cells to the nonpermissive temperature $(C)$. The 0 time point was also treated with $\mathrm{RNaseH}$ and oligo(dT) to indicate the position of the deadenylated mRNA. The quantitative analysis of MFA2 mRNA levels normalized to 7S RNA is graphed for each of the Northern gels. These experiments were repeated between two and five times in these and all subsequent experiments, and error bars represent the variation in RNA levels between individual experiments at each time point.

shutoff. Compared to a sample treated with RNaseH and oligo(dT), which removes the poly(A) tail, we observed a mixed population of MFA2 mRNAs with poly(A) tail lengths from 10 to 12 to $\sim 70$ adenosine nucleotides (nt) (Figs. 1A-D, 2) at time point 0 . This is similar to previously published results (Decker and Parker 1993). In nonstressed cells, the poly(A) tail of MFA2 mRNA shortened at a rate of $\sim 8$ nt per minute, with the entire detectable population reaching an oligo(A) length of $10 \mathrm{nt}$ in $8 \mathrm{~min}$ (Fig. 1, left panels; Fig. 2, dark blue curve). In contrast, in cells exposed to osmotic stress, a significant shortening of the MFA2 poly(A) tail was only detected $15 \mathrm{~min}$ after transcriptional shutoff, and shortening occurred at a slower rate of $2 \mathrm{nt}$ per minute (Fig. 1A, right panel; Fig. 2, light blue curve). A similar inhibition of deadenylation was seen in cells that were exposed to heat stress or glucose deprivation (Fig. 2, green and red curves, respectively). These results show that stress leads to a significant inhibition of deadenylation, which can account for the bulk of the mRNA stabilization observed in response to the stress condition.

\section{Inhibition of deadenylation during stress affects multiple mRNAs}

To determine if the ability of different stresses to induce changes in mRNA turnover is a global effect and not specific to our reporter mRNA, we monitored the decay of the endogenous RPL41A, MFA1, RPS28A, and PGK1 


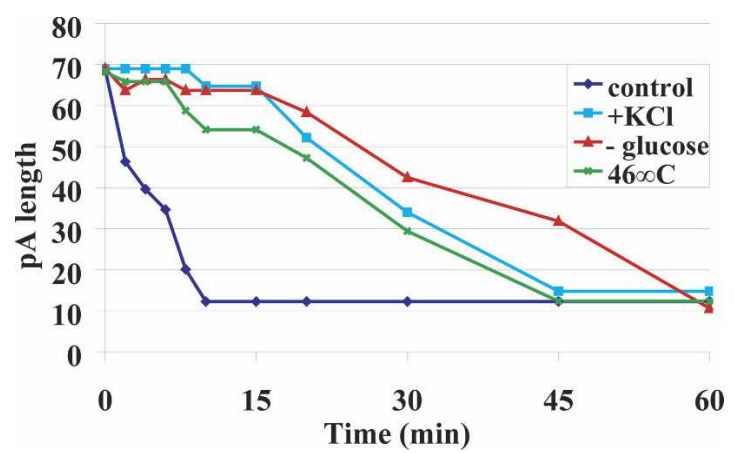

FIGURE 2. Stress inhibits deadenylation of the MFA2 mRNA. Graphic representation of the lengths of the MFA2 mRNA poly(A) tails over time following transcriptional shutoff in control cells and cells exposed to heat shock $\left(46^{\circ} \mathrm{C}\right)$, hyperosmolarity $(\mathrm{KCl} 1 \mathrm{M})$, or glucose deprivation (-glucose). Poly(A) tail lengths were determined by comparison of bands to size standards and the poly(A)-minus mRNA species generated by oligo(dT)-directed $\mathrm{RNaseH}$ cleavage (data not shown).

mRNAs in rpb1-1 cells that were exposed to glucose deprivation or $\mathrm{KCl}$ stress concomitantly with transcriptional shutoff. For both types of stress used, we observed that the decay of multiple mRNAs was inhibited during osmotic stress or glucose deprivation (Fig. 3). Taken together, these observations argue that various types of stress affect turnover of a range of yeast mRNAs.

Analysis of the poly(A) tail lengths of these mRNAs at different time points shows that, similar to the MFA2 mRNA, the poly $(\mathrm{A})$ shortening of various endogenous mRNAs occurs more slowly under stress conditions (Fig. 3; data not shown). Interestingly, some differences in the stress response between individual mRNAs were observed. For example, deadenylation of the MFA1 and RPS28A mRNAs is strongly inhibited following $\mathrm{KCl}$ addition (Fig. 3, middle column), but is only marginally affected by glucose deprivation (Fig. 3, right column). These results suggest that different stresses affect deadenylation slightly differently and/or individual mRNAs may show different responses under some conditions. It should be noted that exposure of cells to $1 \mathrm{M}$ $\mathrm{KCl}$ triggered the strongest inhibition of deadenylation and mRNA decay, and we have used this stress in our subsequent analyses (see below). From these results, we conclude that different stresses are able to trigger a cellular response that causes inhibition of deadenylation and subsequent stabilization of multiple mRNAs. This is in agreement with observations made in mammalian cells, in which several types of stress can cause a global inhibition of mRNA turnover (Gowrishankar et al. 2006).

\section{Inhibition of deadenylation during stress occurs independently of the translation status of the mRNA}

One possible mechanism that could inhibit deadenylation during stress is the protection of mRNAs in subcellular structures that are inaccessible to deadenylases. Indeed, it has been shown that glucose deprivation and hyperosmolarity cause accumulation of yeast mRNAs in cytoplasmic foci called processing bodies (P-bodies) (Sheth and Parker 2003; Teixeira et al. 2005). Further, the major deadenylase Ccr4p is not greatly enriched in these structures during stress (Sheth and Parker 2003; D. Teixeira and R. Parker, unpubl.). In order to determine whether the inhibition of deadenylation is caused by the localization of mRNAs in P-bodies, we treated cells with $0.1 \mathrm{mg} / \mathrm{mL}$ cycloheximide (CYH) simultaneously with exposure to $1 \mathrm{M} \mathrm{KCl}$. Cycloheximide blocks P-body formation even under stress conditions and traps mRNAs in polysomes (Teixeira et al. 2005).

An important result was that stress inhibited deadenylation even in the presence of $\mathrm{CYH}$. In nonstressed cells, the MFA2 mRNA was stabilized in the presence of CYH (half-life $=8 \mathrm{~min}$ ) in comparison to nonstressed cells that were not CYH treated (cf. Fig. 1A, left panel, Fig. 4A, left panel; Fig. 4B, dark blue and brown curves). However, consistent with previous results (Beelman and Parker 1994), cycloheximide does not change the rate of deadenylation of the MFA2 mRNA ( 8 adenosines per minute;
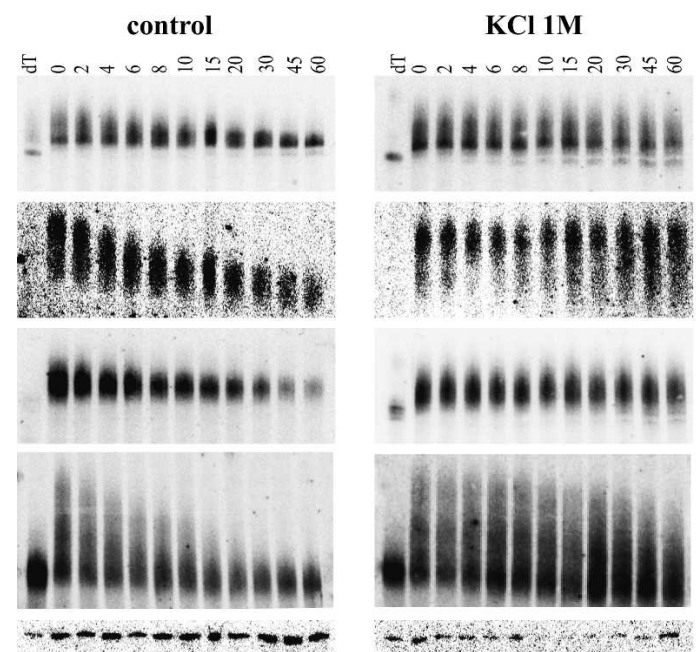

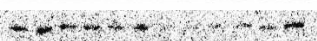
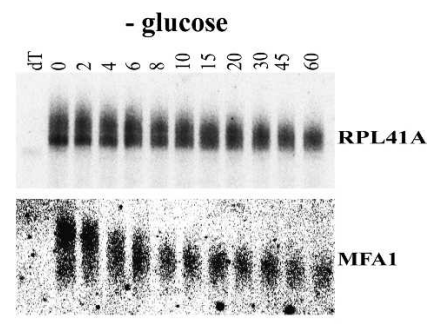

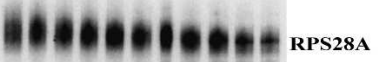

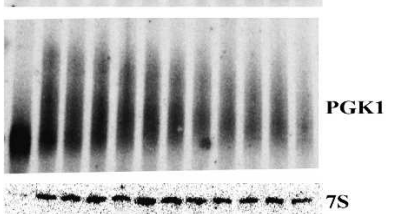

FIGURE 3. Inhibition of deadenylation during stress affects multiple mRNAs. Shown are polyacrylamide Northern gels examining the decay of endogenous RPL41A, MFA1, RPS28A, and PGK1 mRNAs in rpb1-1 strains exposed to osmotic stress $(\mathrm{KCl} 1 \mathrm{M})$ or glucose deprivation (-glucose). Numbers above the lanes represent minutes after transcriptional repression by shifting the cells to the nonpermissive temperature. The 0 time point was also treated with $\mathrm{RNaseH}$ and oligo(dT) to indicate the position of the deadenylated mRNA. To allow for size resolution of the poly(A) tail, the 3' $319 \mathrm{nt}$ of the 1.4-kb PGK1 mRNA were cleaved by oRP70-directed RNaseH cleavage prior to loading on the gel. 
A

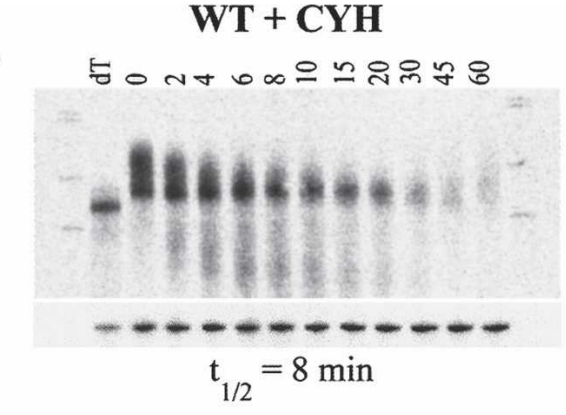

WT + CYH + KCl 1M

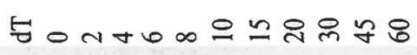

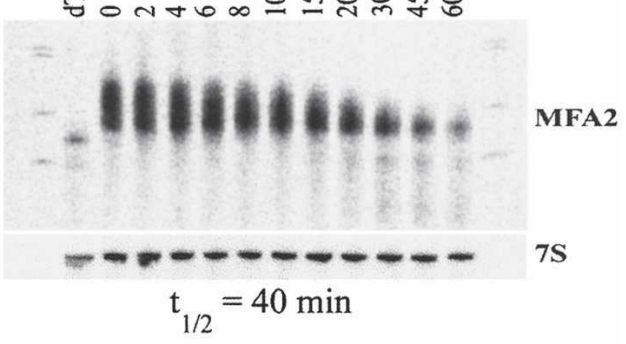

B

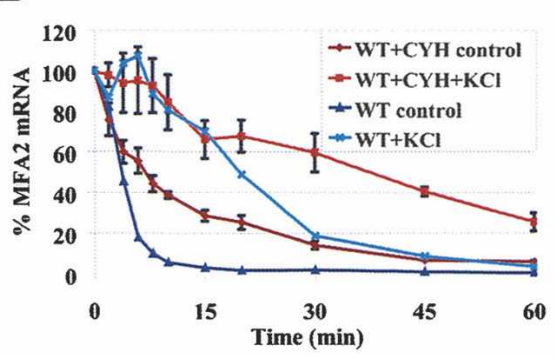

prt1-63

D

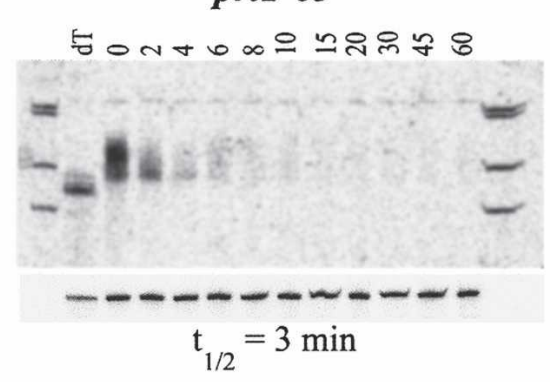

C

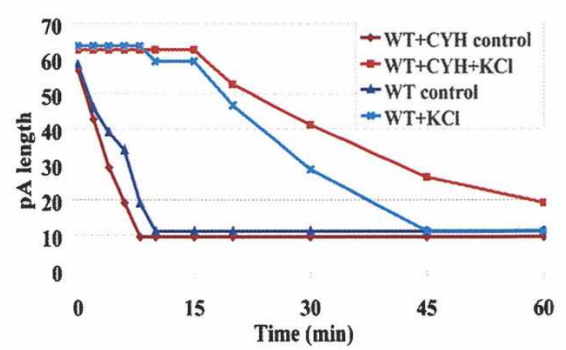

prt1-63+ KCl $1 \mathrm{M}$

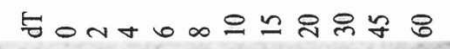

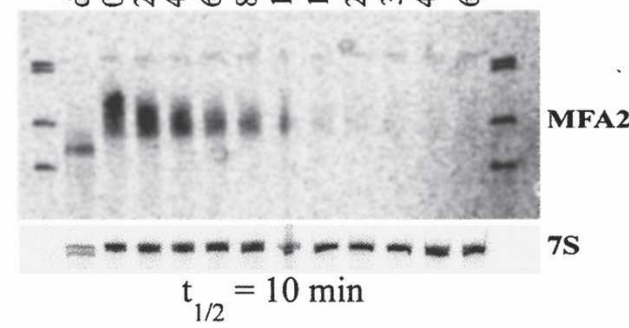

$\mathbf{E}$

F
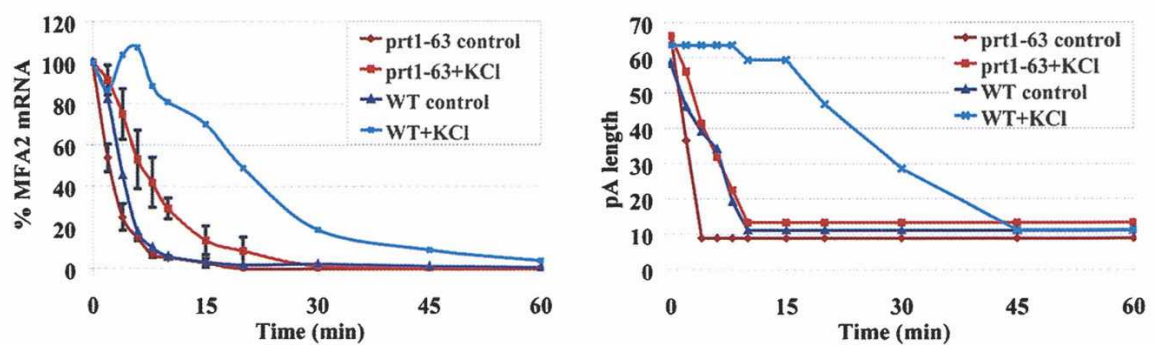

FIGURE 4. Inhibition of deadenylation during stress occurs independent of P-body or polysome localization of the mRNA. ( $A, D)$ Northern gels examining the decay of MFA2 in cycloheximide-treated wild-type cells $(A)$ and in the prt1-63 strain at restrictive temperature $(D)$ without (left panels) and with (right panels) exposure to osmotic stress (KCl $1 \mathrm{M})$. Numbers above the lanes represent minutes after transcriptional repression by shifting the carbon source to glucose. The 0 time point was also treated with $\mathrm{RNaseH}$ and oligo(dT) to indicate the position of the deadenylated mRNA. $(B, E)$ Quantitative analysis of MFA2 mRNA levels normalized to 7S RNA for the Northern gels shown in $A$ and $D$, respectively. ( $C, F)$ Analysis of MFA2 mRNA deadenylation over time for the Northern gels shown in $A$ and $D$, respectively. For comparison, the decay and deadenylation curves obtained for wild-type cells (shown in Figs. 1 and 2) are integrated into the graphs, although for clarity the error bars from those experiments are not repeated.

Fig. 4C, cf. dark blue and brown curves) but instead stabilizes the mRNA by blocking decapping, which can be observed by the persistence of a deadenylated species of the MFA2 mRNA at later time points. Strikingly, when cells were exposed to hyperosmolarity, we observed that deadenylation occurred at a much slower rate than in the nonstressed cells; MFA2 starts to deadenylate $15 \mathrm{~min}$ after stress induction, at the slow rate of $1 \mathrm{nt}$ per minute 
(Fig. 4C, red curve). This demonstrates that stress can inhibit deadenylation even when the mRNA is trapped in polysomes, and therefore the inhibition of deadenylation in response to stress is unlikely to be due to sequestration of transcripts in P-bodies.

The above results argue that the translation status of the mRNA and the inhibition of deadenylation are not mechanistically coupled. To further test this possibility, we examined whether stress could inhibit deadenylation when mRNAs are not translated prior to stress induction. To do this experiment, we used the prt1-63 strain (Schwartz and Parker 1999), which carries a thermolabile allele of an essential component of the eIF3 complex. Upon a shift to the nonpermissive temperature, translation initiation is blocked and mRNAs accumulate in P-bodies under normal as well as under stress conditions (Brengues et al. 2005). Cells were shifted to $38^{\circ} \mathrm{C} 1 \mathrm{~h}$ before exposure to $1 \mathrm{M} \mathrm{KCl}$ to allow inhibition of translation initiation, and $M F A 2 p G$ mRNA decay and deadenylation were then analyzed as previously. To ensure that the temperature shift does not introduce an additional effect on mRNA degradation, a similar temperature shift was performed on wild-type cells with and without concomitant stress induction; the temperature change did not affect MFA2 mRNA decay (data not shown).

In the nonstressed prt1-63 cells, decay and deadenylation of MFA2 mRNA occurred more rapidly than in wildtype cells (cf. Figs. 1A and 4D, left panels; Fig. 4E, cf. dark blue and brown curves). The mRNA half-life is $\sim 3 \mathrm{~min}$ and the MFA2 mRNA deadenylates at a rate of $14 \mathrm{nt}$ per minute in prt1-63 mutants (Fig. 4F, brown curve). This is consistent with previous results showing that when translation initiation is blocked, there is increased deadenylation and decapping of mRNAs (Schwartz and Parker 1999). A significant result was that, under stress conditions, we observed an inhibition of MFA2 mRNA deadenylation and decay in prt1-63 cells (Fig. 4D, cf. right and left panels). The MFA2 half-life (10 min; Fig. 4E, red curve) in prt1-63 mutants is half as long as in stressed wild-type cells, although the relative stabilization of MFA2 mRNA under stress (fourfold) is comparable to the observed stabilization in wild-type cells. Deadenylation occurs immediately after stress exposure in prt1-63 mutants, at a rate of 5-6 nt per minute (Fig. 4F, red curve), and is less inhibited than in wild-type cells. We also observed that stress also inhibited deadenylation in prt1-63 cells when the stress and the shift to $38^{\circ} \mathrm{C}$ were concurrent (data not shown). Therefore, under stress as well as under normal conditions, the MFA2 mRNA is less stable and deadenylates faster in prt1-63 cells than in wild-type cells (see Discussion). These results indicate that stress-induced inhibition of deadenylation is not coupled to cessation of translation, as mRNAs that are already translationally repressed still show slowed deadenylation in response to the stress stimuli.
The observation that stress inhibits deadenylation both in the presence of cycloheximide and when translation initiation is inhibited by the prt1-63 lesion suggests that inhibition of deadenylation during stress can occur, regardless of whether mRNAs are trapped in polysomes or not engaged in translation and thereby accumulating in Pbodies. This indicates that stress-induced inhibition of mRNA deadenylation is not dependent on the translational state or subcytoplasmic localization of the transcript.

\section{Stress inhibits both the Ccr4p/Pop2p/Notp and Pan2p/Pan3p deadenylases}

To gain insight into the mechanisms by which stress inhibits deadenylation, we determined the identity of the deadenylases that are inhibited during stress. The predominant mRNA deadenylase in eukaryotic cells is the Ccr4p/Pop2p/ Notp complex, which is responsible for the majority of the mRNA deadenylation in yeast (Tucker et al. 2001). An additional mRNA deadenylase is the Pan2p/Pan3p complex, which also deadenylates yeast mRNAs, although at a slower rate than the Ccr4p/Pop2p/Notp complex (Tucker et al. 2001). To determine whether one or both of these deadenylases were inhibited during stress, we examined the deadenylation of the $M F A 2 p G$ reporter in strains lacking a functional Ccr4p/Pop2p/Notp or Pan2p/Pan3p complex. The logic of this experiment is that the analysis of deadenylation in strains with only one functional deadenylation complex would help to directly determine the effect of stress on that specific deadenylase.

We first examined a pan $2 \Delta$ strain, in which deadenylation is only due to the Ccr4p/Pop2p/Notp complex (Tucker et al. 2001). In nonstressed pan $2 \Delta$ mutants, MFA2 mRNA decay and deadenylation do not differ significantly from those observed in wild-type cells, consistent with the fact that $\operatorname{Pan} 2 \mathrm{p} / \operatorname{Pan} 3 \mathrm{p}$ represents the minor yeast deadenylase under normal conditions (Fig. 5A, left panel; Fig. 5B, cf. dark blue and brown curves). In contrast, in stressed pan $2 \Delta$ cells, the MFA2pG mRNA half-life is prolonged (30 min, sixfold stabilization; Fig. 5A, right panel; Fig. 5B, cf. light blue and red curves) compared to wild-type cells (fourfold stabilization), and deadenylation occurs much more slowly (Fig. 5A, cf. right and left panels). This inhibition of deadenylation in the pan $2 \Delta$ strain, in which the Ccr4p/ Pop2p/Notp complex is the only functional deadenylase, demonstrates that the ability of Ccr4p/Pop $2 \mathrm{p} /$ Notp to deadenylate mRNAs is inhibited during stress.

We next examined a $c c r 4 \Delta$ strain, in which the residual deadenylation is solely due to the Pan $2 \mathrm{p} / \mathrm{Pan} 3 \mathrm{p}$ complex (Tucker et al. 2001). In agreement with previous reports (Tucker et al. 2001), the MFA2pG mRNA in the nonstressed ccr $4 \Delta$ cells is stabilized twofold (Fig. 5C, left panel; Fig. 5D, brown curve: half-life $=10 \mathrm{~min}$ ) compared to the wild-type strain, and deadenylation occurs at a slower rate of $2 \mathrm{nt}$ per minute, stopping with a residual poly(A) tail of 20-25 

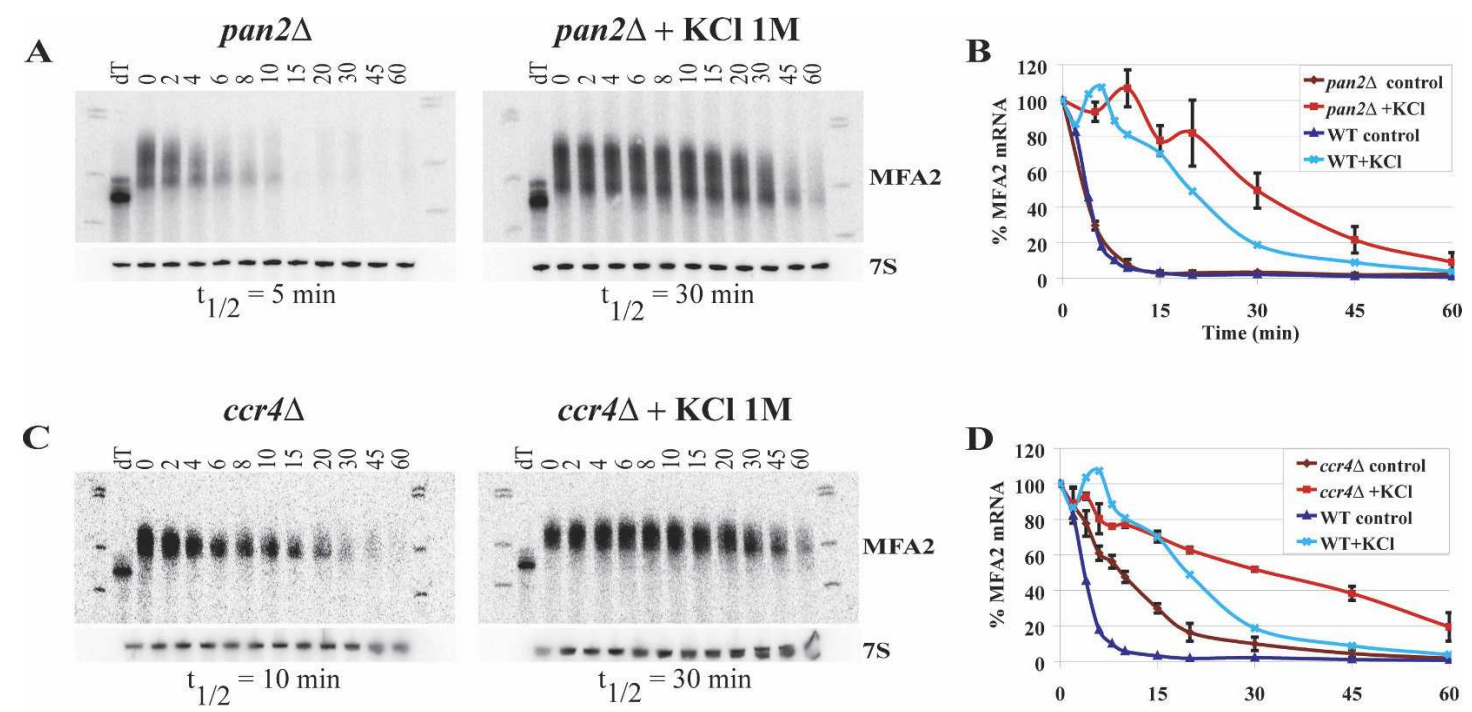

FIGURE 5. Stress inhibits both the Ccr4p/Pop2p/Notp and Pan2p/Pan3p deadenylases. $(A, C)$ Northern gels examining the decay of $M F A 2$ mRNA in $c c r 4 \Delta(A)$ and pan $2 \Delta(C)$ mutants without (left panels) and with (right panels) exposure to osmotic stress ( $\mathrm{KCl} 1 \mathrm{M})$. Numbers above the lanes represent minutes after transcriptional repression by shifting the carbon source to glucose. The 0 time point was also treated with $\mathrm{RNaseH}$ and oligo(dT) to indicate the position of the deadenylated mRNA. (B,D) Quantitative analysis of MFA2 mRNA levels normalized to 7S RNA for the Northern gels shown in $A$ and $C$, respectively. For comparison, the decay curves obtained for wild-type cells (shown in Figs. 1 and 3 ) are integrated into the graphs, although for clarity the error bars from those experiments are not repeated.

adenosines. However, an important result was that stress still caused an inhibition of deadenylation and a stabilization of the MFA2pG mRNA (half-life $=30 \mathrm{~min}$; Fig. 5C, right panel; Fig. 5D, red curve). The inhibition of deadenylation and decay in the $c c r 4 \Delta$ strain, where the Pan $2 \mathrm{p} / \mathrm{Pan} 3 \mathrm{p}$ complex is the only functional deadenylase, demonstrates that the ability of the Pan2p/Pan3p complex to deadenylate mRNAs is inhibited during stress. It should be noted that the difference in deadenylation rates with and without stress in the $c c r 4 \Delta$ strain are not as severe as in wild-type cells due to the fact that Ccr4p is the predominant nuclease. Taken together, we interpret these observations to indicate that both the Ccr4p/Pop2p/Notp and the Pan2p/Pan3p deadenylases can be inhibited during stress, although the largest effect is on the function of the Ccr4p/Pop2p/Notp complex.

In both $c c r 4 \Delta$ and pan $2 \Delta$ mutant strains, deadenylation is inhibited during stress, but still occurs to some extent. There are two possible explanations for this residual deadenylation seen during stress. First, the function of both the Ccr4p/Pop2p/Notp and Pan2p/Pan3p deadenylases might be partially impaired in response to stress, and their residual activity accounts for the observed slow deadenylation during stress. Alternatively, the ongoing deadenylation during stress can be explained by complete inhibition of the function of both the Ccr4p/Pop $2 p /$ Notp and $\operatorname{Pan} 2 \mathrm{p} / \mathrm{Pan} 3 \mathrm{p}$ deadenylases and the activation of a stress-specific deadenylase.

We performed two experiments to examine if a stressspecific deadenylase was responsible for the residual deadenylation seen during stress. First, we tested whether the cytoplasmic exosome, a major $3^{\prime}$ to $5^{\prime}$ exonuclease, was required for the residual deadenylation during stress by examining deadenylation in ski2 $\Delta$ strains. Ski2p is a component of the Ski complex that associates with the cytoplasmic exosome and is essential for the exosome's cytoplasmic function (Anderson and Parker 1998). In ski2d mutants, the decay and deadenylation rates of the MFA2 mRNA did not differ from those observed in wild-type cells, under both normal and stress conditions (data not shown). Thus, the cytoplasmic exosome is not responsible for the residual deadenylation that occurs during stress. Second, we asked if an unknown deadenylase is activated during stress. Here, we examined the deadenylation and decay of the MFA2 mRNA in a $c c r 4 \operatorname{pan} 2 \Delta$ strain, which has no measurable deadenylation activity under normal growth conditions (Tucker et al. 2001). We observed no deadenylation of the $M F A 2 p G$ reporter $m R N A$ in the $c c r 4 \Delta$ pan $2 \Delta$ strain either with or without stress (Fig. 6A). Thus, no other nuclease seems to be involved in mRNA deadenylation during normal or stress conditions.

Analysis of MFA2 mRNA decay in the $c c r 4 \Delta$ pan $2 \Delta$ mutant strain revealed an $\sim 1$.4-fold increase in mRNA half-life with versus without stress induction (Fig. 6A, B, cf. brown and red curves). Though this stabilization is significant, it is less than what was observed in wild-type cells (four- to fivefold). This difference suggests that stress might also inhibit mRNA decay downstream of deadenylation, presumably at decapping or $5^{\prime}$ to $3^{\prime}$ exonuclease digestion. To confirm this, we examined mRNA decay rates in $c c r 4 \Delta$ pan $2 \Delta$ cells in which decapping was inhibited by cycloheximide treatment. Under these 

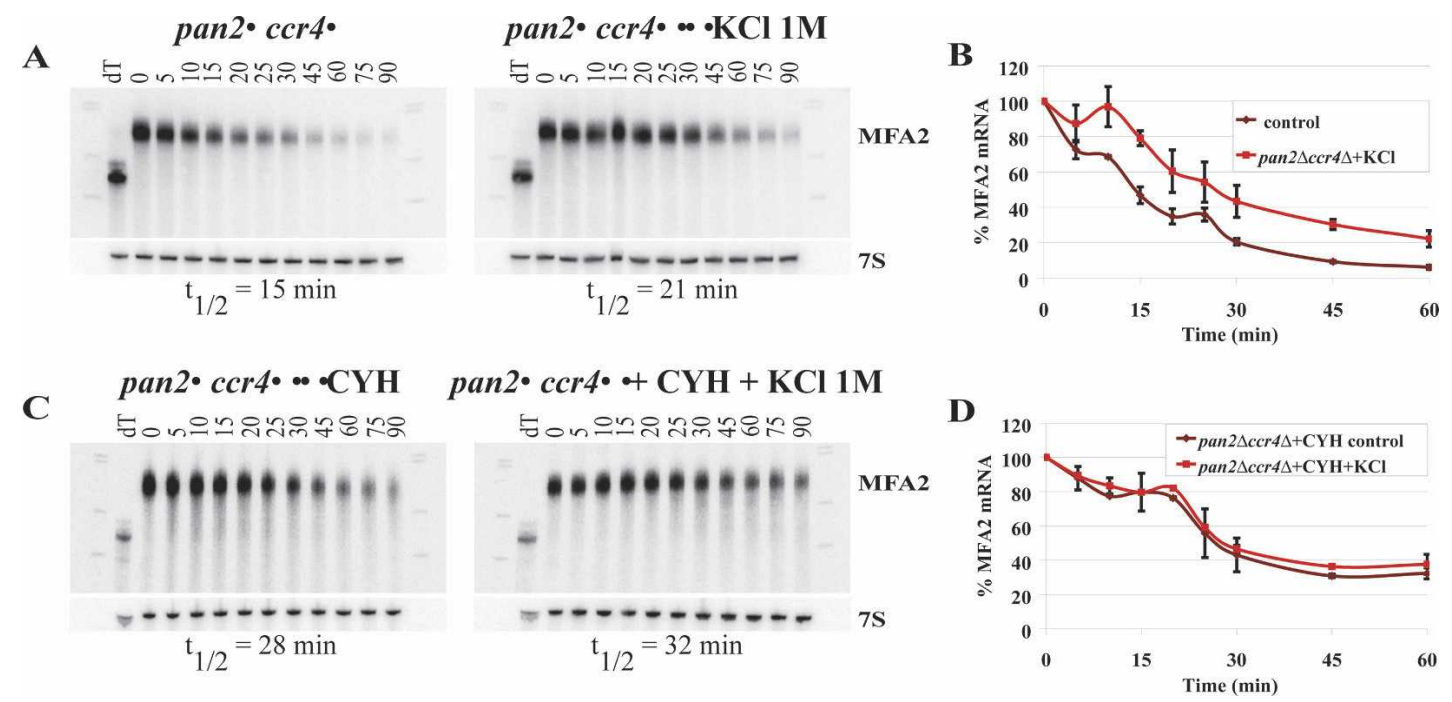

FIGURE 6. Stress affects deadenylation and, to a lesser extent, $5^{\prime}$ to $3^{\prime}$ degradation. $(A, C)$ Northern gels examining the decay of $M F A 2$ mRNA in pan $2 \Delta c c r 4 \Delta(A)$ and cycloheximide-treated pan $2 \Delta c c r 4 \Delta(C)$ mutants without (left panels) and with (right panels) exposure to osmotic stress $(\mathrm{KCl} 1 \mathrm{M})$. Numbers above the lanes represent minutes after transcriptional repression by shifting the carbon source to glucose. The 0 time point was also treated with RNaseH and oligo $(\mathrm{dT})$ to indicate the position of the deadenylated mRNA. (B,D) Quantitative analysis of MFA2 mRNA levels normalized to 7S RNA for the Northern gels shown in $A$ and $C$, respectively.

conditions, the MFA2 mRNA showed similar decay rates in the absence and presence of stress (Fig. 6C, D, cf. brown and red curves). This suggests that stress can also inhibit, to some extent, either decapping or $5^{\prime}$ to $3^{\prime}$ degradation, which could be consistent with previous observations that amino acid starvation can inhibit $5^{\prime}$ to $3^{\prime}$ exonuclease activity (Bénard 2004).

\section{DISCUSSION}

In this work we demonstrate that multiple stresses inhibit the deadenylation of yeast mRNAs and thereby lead to their stabilization. The key observation is that direct measurement of the deadenylation and mRNA decay rates of the MFA2 mRNA indicates that deadenylation and degradation are inhibited by hyperosmolarity, heat shock, and glucose deprivation (Figs. 1, 2). This inhibition of deadenylation appears to be broad and target both unstable and stable transcripts since the deadenylation and turnover of the PGK1, MFA1, RPL41A, and RPS28A mRNAs are also inhibited by stress (Fig. 3). These results complement previous studies that have shown that in yeast, general mRNA turnover is slowed during amino acid starvation, salt stress, or sugar-induced osmotic stress, although whether deadenylation was affected in those cases was not examined (Bénard 2004; Greatrix and van Vuuren 2006; Todeschini et al. 2006). Moreover, heat shock has also been reported to stabilize ARE-containing mRNAs in mammalian cells and to affect the decay and deadenylation of the Hsp70 mRNA in Drosophila (Dellavalle et al. 1994; Laroia et al. 1999; Zhao et al. 2002). Importantly, recent observations in HeLa cells demonstrate that several stresses, including UV-B light, hydrogen peroxide, heat shock, and hyperosmolarity, inhibited mRNA deadenylation and decay (Gowrishankar et al. 2006). Thus, deadenylation is inhibited by a number of stresses in both yeast and metazoans and therefore appears to be a common and conserved aspect of at least some stress responses.

Several observations argue that the inhibition of deadenylation by osmotic stress occurs solely at a post-translational level and is independent of new transcription or translation. First, osmotic stress strongly inhibits deadenylation of the MFA2 mRNA even when general transcription has been inhibited by inactivation of RNA polymerase II (Fig. 1). Similarly, osmotic stress strongly inhibits deadenylation when new translation is inhibited by the addition of cycloheximide (Fig. 4). The possibility of post-translational regulation is further strengthened by the observation that the inhibition of deadenylation is extremely rapid and occurs within 2 min of exposure to the stress stimuli (Fig. 1).

Analysis of various mutant strains indicates that osmotic stress inhibits the function of both the Ccr4p/Pop2p/Notp complex, which is the major cytoplasmic mRNA, as well as the minor Pan2p/Pan3p deadenylase. Specifically, in pan $2 \Delta$ strains, where mRNA deadenylation occurs solely by the Ccr4p/Pop2p/Notp complex (Tucker et al. 2001), osmotic stress still strongly inhibits deadenylation and mRNA decay (Fig. 5). This demonstrates that Ccr4p-driven deadenylation can be inhibited by osmotic stress. Similarly, in $c c r 4 \Delta$ strains, in which mRNA deadenylation occurs solely by the Pan2p/Pan3p complex, albeit at reduced efficiency (Tucker et al. 2001), osmotic stress still inhibits deadenylation and mRNA decay (Fig. 5). The ability of stress to inhibit both cytoplasmic deadenylases suggests either that both these 
deadenylases are targets of inactivating modifications during stress or that stress is altering the properties of the mRNP substrate such that the poly(A) tail is no longer available for deadenylation by either enzyme.

Because the stresses inhibiting deadenylation are also known to inhibit translation initiation and lead to mRNAs accumulating in P-bodies, one could propose that sequestration of mRNAs into P-bodies might protect their poly(A) tails from deadenylation. However, this possibility is extremely unlikely because deadenylation is inhibited even in the presence of cycloheximide, which traps mRNAs in polysomes and prevents P-body formation (Sheth and Parker 2003; Teixeira et al. 2005). This suggests that the inhibition of deadenylation is independent of the translation status of the mRNA. Consistent with that interpretation, we observed that osmotic stress also inhibited deadenylation in cells where translation initiation was further compromised by the prt1-63 mutation, which carries a defect in a subunit of the eIF3 translation initiation factor. It should be noted that compared to similar conditions for wild-type strains, deadenylation and decay occurred faster in the prt1-63 strain even in the presence of stress. This suggests that either the residual translation that occurs in wild-type strains with stress can also inhibit deadenylation to some extent (Schwartz and Parker 1999) or that the prt1-63 lesion might trap the mRNA in a biochemical state wherein the poly(A) tail is more susceptible to deadenylation. Nevertheless, the fact that deadenylation and decay can be inhibited in response to osmotic stress when transcripts are localized in polysomes or in P-bodies demonstrates that this control occurs independently of the translational state of the transcript.

Based on these results, two possible models can be proposed for how stress leads to an inhibition of deadenylation. First, different stresses could lead to post-translational modifications of both the Ccr4p/Pop2p/Notp and Pan2p/Pan3p complexes, thereby inactivating their function and inhibiting deadenylation. Alternatively, different stresses might lead to an alteration in the mRNP composition such that the poly(A) is less available for deadenylation. For example, the stress response might trigger modifications of the binding properties of various proteins such that the poly(A) binding protein (Pablp), which is normally associated with the $\operatorname{poly}(\mathrm{A})$ tail, was replaced with an alternative RNA binding protein. Since the Ccr4p/Pop2p/Notp complex is inhibited by proteins bound to the poly(A) tail (Tucker et al. 2002), and the Pan2p/Pan3p complex is stimulated by Pablp on the poly(A) (Boeck et al.
1996), such a novel poly(A) binding protein could thereby inhibit both deadenylases. Interestingly, it should be noted that if stress inhibits deadenylation by promoting a specific mRNP complex formation, then it is likely that such a complex is also stabilized or destabilized in an mRNAspecific manner in the absence of stress to control deadenylation rates on individual mRNAs. Future work will be required to determine which of these possible mechanisms underlies the control of deadenylation.

Our results have implications for understanding other observations in the literature. For instance, the stressinduced inhibition of deadenylation in mammalian cells (Gowrishankar et al. 2006) could share common mechanisms with the regulation process in yeast and involve the inhibition of both the Ccr4p/Pop2p/Notp and Pan2p/Pan3p complexes. Moreover, we suggest that inhibition of deadenylation during stress could be important for the translational reactivation of miRNA-repressed mRNAs in response to cellular stresses (Bhattacharyya et al. 2006). This hypothesis is based on the fact that the Ccr4p/Pop2p/Notp complex has been recently shown to be important for miRNA-induced deadenylation (Behm-Ansmant et al. 2006). Thus, the stress-induced inhibition of the deadenylation activity of the Ccr4p/Pop2p/ Notp complex could participate in the translational derepression of microRNA targets under conditions where global translation is inhibited.

The inhibition of deadenylation and mRNA decay during stress is likely to play an important role in an integrated cellular response to stress. Many cellular stresses lead to an inhibition of translation initiation and the sequestration of the mRNAs into cytoplasmic P-bodies or stress granules (for review, see Anderson and Kedersha 2006; Bond 2006), presumably to allow preferential translation of newly synthesized mRNAs that encode proteins that alleviate the
TABLE 1. Strains used in this study

\begin{tabular}{|c|c|c|}
\hline Strain & Genotype & Reference \\
\hline yRP840 & $\begin{array}{l}\text { MATa his4-539 leu2-3,112 trp1-1 } \\
\quad \text { ura3-52 cup1::LEU2/PGK1pG/MFA2pG }\end{array}$ & (Hatfield et al. 1996) \\
\hline yRP1616 & $\begin{array}{l}\text { MATa his4-539 leu2-3,112 trp1-1 } \\
\text { ura3-52 cup1::LEU2/PGK1pG/ } \\
\text { MFA2pG cCr4::NEO }\end{array}$ & (Tucker et al. 2001) \\
\hline yRP1619 & $\begin{array}{l}\text { MATa his4-539 leu2-3,112 trp1-1 } \\
\text { ura3-52 cup1::LEU2/PGK1pG/ } \\
\text { MFA2pG pan2::URA3 }\end{array}$ & (Tucker et al. 2001) \\
\hline yRP1620 & $\begin{array}{l}\text { MATa his4-539 leu2-3,112 trp1-1 } \\
\text { ura3-52 cup1::LEU2/PGK1pG/ } \\
\text { MFA2pG cCr4::NEO pan2::URA3 }\end{array}$ & (Tucker et al. 2001) \\
\hline yRP1195 & $\begin{array}{l}\text { MATa his4-539 leu2-3,112 trp1-1 } \\
\text { ura3-52 cup1::LEU2/PGK1pG/ } \\
\text { MFA2pG ski2::LEU2 }\end{array}$ & (Anderson and Parker 1998) \\
\hline yRP1326 & $\begin{array}{l}\text { MAT } \boldsymbol{\alpha} \text { leu2-3,112 trp1 ura3-52 prt1-63 } \\
\quad \text { cup1::LEU2/PGK1pG/MFA2pG }\end{array}$ & (Schwartz and Parker 1999) \\
\hline yRP582 & MATa rpb1-1 leu2-3,112 ura3-52 & (Muhlrad et al. 1994) \\
\hline
\end{tabular}


stress condition. However, because P-bodies can be sites of mRNA degradation, the accumulation of mRNAs within these structures might have been expected to enhance mRNA degradation. Thus, by inhibiting deadenylation during such stresses, the transcripts can accumulate in P-bodies as adenylated mRNAs, which are unable to undergo decapping and further degradation. This allows the transcripts to persist until the cell recovers from stress and then to exit P-bodies and reenter the translating pool, a process that has now been demonstrated in both yeast and mammalian cells (Brengues et al. 2005; Bhattacharyya et al. 2006). Thus, the inhibition of deadenylation by cellular stress allows the maintenance of the current transcriptome for translation after stress recovery.

\section{MATERIAL AND METHODS}

\section{Yeast strains}

The genotypes of all the strains (Saccharomyces cerevisiae) used in this study are listed in Table 1 . Strains were grown in standard media. With the exception of yRP582, all strains carry an MFA2pG reporter cassette driven by the GAL1 upstream activating sequence (GAL1 UAS), genomically integrated at the CUP1 locus.

\section{mRNA decay measurements, RNA isolation, and RNA analysis}

Steady-state transcriptional shutoff experiments were performed as previously described (Caponigro et al. 1993), with slight modifications. All experiments were repeated between two and five times and the error bars in each figure represent the variation in the RNA levels at each time point in different experiments. Thermal repression of transcription (yRP582) was accomplished by growing cells until mid-log phase at $24^{\circ} \mathrm{C}$, pelleting the cells, and then resuspending them in medium prewarmed to $38^{\circ} \mathrm{C}$. Glucose repression (yRP840) was carried out by growing cells in medium containing $2 \%$ galactose until mid-log phase, pelleting the cells, and then resuspending them in medium containing only $2 \%$ glucose as a carbon source. Repression of translation prior to shutoff (yRP1326) was performed by growing cells in medium containing $2 \%$ galactose until early $\log$ phase at $24^{\circ} \mathrm{C}$, pelleting the cells, and resuspending them in medium prewarmed to $38^{\circ} \mathrm{C} 1 \mathrm{~h}$ before glucose-induced repression of transcription. Cells were exposed to stress simultaneously with transcriptional shutoff. In all strains except yRP582, the stress response was induced by adding either 1 $\mathrm{M}$ potassium chloride $(\mathrm{KCl})$ or $5 \mathrm{mM}$ dithiothreitol (DTT) to the resuspension media or by resuspending the cells in medium prewarmed to $46^{\circ} \mathrm{C}$. For the experiments using the yRP582 strain, induction of stress was performed by resuspending the cells in media lacking a carbon source. An aliquot of the cells was then removed at each time point after shutoff and the mRNA was isolated as described previously (Caponigro et al. 1993). RNaseH and polyacrylamide Northern assays and analysis of mRNA poly(A) tail lengths were carried out as previously (Muhlrad and Parker 1992). Deadenylation rates were determined by measuring the lengths of the longest poly(A) tail lengths at each time point and plotting their decrease in length over time. Transcript levels were quantified on a PhosphorImager (Amersham Biosciences) and normalized to $7 \mathrm{~S}$ RNA, a polymerase III transcript (Caponigro et al. 1993).

\section{ACKNOWLEDGMENTS}

We thank the members of the Parker laboratory for helpful discussions and critical reading of the manuscript. This work was supported by the Howard Hughes Medical Institute and grant GM45443 from the National Institute of Health. Daniela Teixeira was supported by Fundacao para a Ciencia e Tecnologia (SFRH/ BD/2739/2000), Portugal.

Received July 14, 2006; accepted July 28, 2006.

\section{REFERENCES}

Anderson, P. and Kedersha, N. 2006. RNA granules. J. Cell Biol. 172: 803-808.

Anderson, J.S. and Parker, R. 1998. The $3^{\prime}$ to $5^{\prime}$ degradation of yeast mRNAs is a general mechanism for mRNA turnover that requires the SKI2 DEVH box protein and $3^{\prime}$ to $5^{\prime}$ exonucleases of the exosome complex. EMBO J. 17: 1497-1506.

Beelman, C.A. and Parker, R. 1994. Differential effects of translational inhibition in cis and in trans on the decay of the unstable yeast MFA2 mRNA. J. Biol. Chem. 269: 9687-9692.

Behm-Ansmant, I., Rehwinkel, J., Doerks, T., Stark, A., Bork, P., and Izaurralde, E. 2006. mRNA degradation by miRNAs and GW182 requires both CCR4:NOT deadenylase and DCP1:DCP2 decapping complexes. Genes \& Dev 20: 1885-1898.

Bénard, L. 2004. Inhibition of 5' to $3^{\prime}$ mRNA degradation under stress conditions in Saccharomyces cerevisiae: From GCN4 to MET16. RNA 10: 458-468.

Bhattacharyya, S.N., Habermacher, R., Martine, U., Closs, E.I., and Filipowicz, W. 2006. Relief of microRNA-mediated translational repression in human cells subjected to stress. Cell 125: 1111-1124.

Boeck, R., Tarun Jr., S., Rieger, M., Deardorff, J.A., Müller-Auer, S., and Sachs, A.B. 1996. The yeast Pan2 protein is required for poly(A)-binding protein-stimulated poly(A)-nuclease activity. $J$. Biol. Chem. 271: 432-438.

Bond, U. 2006. Stressed out! Effects of environmental stress on mRNA metabolism. FEMS Yeast Res. 6: 160-170.

Brengues, M., Teixeira, D., and Parker, R. 2005. Movement of eukaryotic mRNAs between polysomes and cytoplasmic processing bodies. Science 310: 486-489.

Caponigro, G., Muhlrad, D., and Parker, R. 1993. A small segment of the MAT $\alpha 1$ transcript promotes mRNA decay in Saccharomyces cerevisiae: A stimulatory role for rare codons. Mol. Cell. Biol. 13: $5141-5148$

Coller, J. and Parker, R. 2005. General translational repression by activators of mRNA decapping. Cell 122: 875-886.

Cougot, N., Babajko, S., and Séraphin, B. 2004. Cytoplasmic foci are sites of mRNA decay in human cells. J. Cell Biol. 165: 31-40.

Daugeron, M.C., Mauxion, F., and Séraphin, B. 2001. The yeast POP2 gene encodes a nuclease involved in mRNA deadenylation. Nucleic Acids Res. 29: 2448-2455.

Decker, C.J. and Parker, R. 1993. A turnover pathway for both stable and unstable mRNAs in yeast: Evidence for a requirement for deadenylation. Genes \& Dev. 7: 1632-1643.

Dellavalle, R.P., Petersen, R., and Lindquist, S. 1994. Preferential deadenylation of Hsp70 mRNA plays a key role in regulating Hsp70 expression in Drosophila melanogaster. Mol. Cell. Biol. 14: 3646-3659.

Gowrishankar, G., Winzen, R., Dittrich-Breiholz, O., Redich, N., Kracht, M., and Holtmann, H. 2006. Inhibition of mRNA deadenylation and degradation by different types of cell stress. Biol. Chem. 387: 323-327. 
Greatrix, B.W. and van Vuuren, H.J. 2006. Expression of the HXT13, HXT15 and HXT17 genes in Saccharomyces cerevisiae and stabilization of the HXT1 gene transcript by sugar-induced osmotic stress. Curr. Genet. 49: 205-217.

Hatfield, L., Beelman, C.A., Stevens, A., and Parker, R. 1996. Mutations in trans-acting factors affecting mRNA decapping in Saccharomyces cerevisiae. Mol. Cell. Biol. 16: 5830-5838.

Herrick, D., Parker, R., and Jacobson, A. 1990. Identification and comparison of stable and unstable mRNAs in Saccharomyces cerevisiae. Mol. Cell. Biol. 10: 2269-2284.

Herruer, M.H., Mager, W.H., Raué, H.A., Vreken, P., Wilms, E., and Planta, R.J. 1988. Mild temperature shock affects transcription of yeast ribosomal protein genes as well as the stability of their mRNAs. Nucleic Acids Res. 16: 7917-7929.

Holcik, M. and Sonenberg, N. 2005. Translational control in stress and apoptosis. Nat. Rev. Mol. Cell Biol. 6: 318-327.

Jona, G., Choder, M., and Gileadi, O. 2000. Glucose starvation induces a drastic reduction in the rates of both transcription and degradation of mRNA in yeast. Biochim. Biophys. Acta 1491: $37-48$.

Laroia, G., Cuesta, R., Brewer, G., and Schneider, R.J. 1999. Control of mRNA decay by heat shock-ubiquitin-proteasome pathway. Science 284: 499-502.

Liu, J., Rivas, F.V., Wohlschlegel, J., Yates 3rd, J.R., Parker, R., and Hannon, G.J. 2005. A role for the P-body component GW182 in microRNA function. Nat. Cell Biol. 7: 1261-1266.

Muhlrad, D. and Parker, R. 1992. Mutations affecting stability and deadenylation of the yeast MFA2 transcript. Genes \& Dev. 6: 21002111.

Muhlrad, D., Decker, C.J., and Parker, R. 1994. Deadenylation of the unstable mRNA encoded by the yeast MFA2 gene leads to decapping followed by $5^{\prime} \rightarrow 3^{\prime}$ digestion of the transcript. Genes \& Dev. 8: 855-866.

Parker, R. and Song, H. 2004. The enzymes and control of eukaryotic mRNA turnover. Nat. Struct. Mol. Biol. 11: 121-127.
Schwartz, D.C. and Parker, R. 1999. Mutations in translation initiation factors lead to increased rates of deadenylation and decapping of mRNAs in Saccharomyces cerevisiae. Mol. Cell. Biol. 19: 5247-5256.

Sen, G.L. and Blau, H.M. 2005. Argonaute 2/RISC resides in sites of mammalian mRNA decay known as cytoplasmic bodies. Nat. Cell Biol. 7: 633-636.

Sheth, U. and Parker, R. 2003. Decapping and decay of messenger RNA occur in cytoplasmic processing bodies. Science 300: 805-808.

Teixeira, D., Sheth, U., Valencia-Sanchez, M.A., Brengues, M., and Parker, R. 2005. Processing bodies require RNA for assembly and contain nontranslating mRNAs. RNA 11: 371-382.

Todeschini, A.L., Condon, C., and Bénard, L. 2006. Sodium-induced GCN4 expression controls the accumulation of the $5^{\prime}$ to $3^{\prime}$ RNA degradation inhibitor, $3^{\prime}$-phosphoadenosine $5^{\prime}$-phosphate. J. Biol. Chem. 281: 3276-3282.

Travers, K.J., Patil, C.K., Wodicka, L., Lockhart, D.J., Weissman, J.S., and Walter, P. 2000. Functional and genomic analyses reveal an essential coordination between the unfolded protein response and ER-associated degradation. Cell 101: 249-258.

Tucker, M., Valencia-Sanchez, M.A., Staples, R.R., Chen, J., Denis, C.L., and Parker, R. 2001. The transcription factor associated Ccr4 and Caf1 proteins are components of the major cytoplasmic mRNA deadenylase in Saccharomyces cerevisiae. Cell 104: $377-386$.

Tucker, M., Staples, R.R., Valencia-Sanchez, M.A., Muhlrad, D., and Parker, R. 2002. Ccr4p is the catalytic subunit of a Ccr4p/Pop2p/ Notp mRNA deadenylase complex in Saccharomyces cerevisiae. EMBO J. 21: 1427-1436.

Zhao, M., Tang, D., Lechpammer, S., Hoffman, A., Asea, A., Stevenson, M.A., and Calderwood, S.K. 2002. Double-stranded RNA-dependent Protein Knase (pkr) is essential for thermotolerance, accumulation of HSP70, and stabilization of ARE-containing HSP70 mRNA during stress. J. Biol. Chem. 277: 4453944547. 

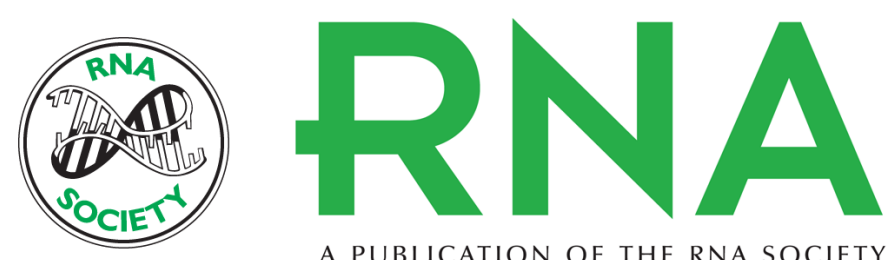

A PUBLICATION OF THE RNA SOCIETY

\section{Translation-independent inhibition of mRNA deadenylation during stress in Saccharomyces cerevisiae}

Valérie Hilgers, Daniela Teixeira and Roy Parker

RNA 2006 12: 1835-1845

References This article cites 36 articles, 22 of which can be accessed free at:

http://rnajournal.cshlp.org/content/12/10/1835.full.html\#ref-list-1

License

Email Alerting Receive free email alerts when new articles cite this article - sign up in the box at the Service top right corner of the article or click here.

To subscribe to RNA go to:

http://rnajournal.cshlp.org/subscriptions 